\title{
ELECCIONES Y RECHAZOS ENTRE PARES EN SITUACIONES DE TRABAJO GRUPAL MEDIADO TECNOLÓGICAMENTE
}

\author{
(SELECTION AND PEER REJECTION IN \\ TECHNOLOGICALLY-MEDIATED GROUP WORK)
}

\author{
Analía Claudia Chiecher Costa \\ Consejo Nacional de Investigaciones Científicas y Tecnológicas y Universidad \\ Nacional de Río Cuarto, Argentina
}

\section{RESUMEN}

El escrito atiende a algunos aspectos vinculados con la dimensión social de las interacciones en grupos de alumnos que resuelven una tarea académica en entorno virtual. Participaron del estudio 154 estudiantes distribuidos en 36 grupos, quienes una vez finalizado el trabajo grupal, respondieron un cuestionario sociométrico. Los resultados describen características de los grupos, informan sobre algunos índices sociométricos y describen razones de elección y rechazo. La discusión destaca como importantes las habilidades de co-regulación del aprendizaje en entornos virtuales.

Palabras clave: entornos virtuales, trabajo en grupos, interacciones entre pares, nominaciones positivas, rechazos.

\begin{abstract}
This paper addresses certain aspects linked to the social dimension of student interactions that occur during an academic activity in a virtual learning environment. 154 students, who were divided into 36 groups, participated in this study by responding to a sociometric test. The results show the group characteristics and provide an anslysis of some of the sociometric values, as well as the reasons for selection and rejection of certain factors. The discussion highlights the importance of developing the skills of co-regulated learning in virtual environments.
\end{abstract}

Keywords: virtual environments, group work, peer interactions, positive nominations, rejections.

La mayoría de los estudios sobre aprendizaje colaborativo online han focalizado en variables relacionadas con la construcción del conocimiento, siendo menos estudiado el funcionamiento de los grupos desde un punto de vista interpersonal o social.

Atendiendo a la novedad del tema y a la escasez de estudios realizados al respecto, interesa en este artículo analizar aspectos vinculados con las relaciones 
interpersonales, aceptaciones y rechazos que se generan y despliegan en grupos de alumnos que trabajan colaborativamente en contextos virtuales.

Teóricamente, las actividades de aprendizaje diseñadas en formatos colaborativos deberían favorecer el intercambio socio-cognitivo entre los estudiantes, incrementar la motivación y contribuir a elevar la calidad del conocimiento que se construye conjuntamente. De igual modo, las tareas académicas pensadas para ser resueltas entre pares constituirían un elemento clave en entornos 'poderosos' para el aprendizaje (de Jong y Pieters, 2006; Rinaudo, en prensa). Esto es, resulta entonces muy deseable que en contextos virtuales se propongan tareas a ser abordadas en pequeños grupos de alumnos. No obstante, en la práctica no siempre se constatan los beneficios de la cooperación -o trabajo grupal- sobre el aprendizaje. Siguiendo a López y Álvarez (2011), una de las causas de este hecho puede estar relacionada con las dificultades de los estudiantes para regular (y co-regular) sus comportamientos y emociones cuando participan en situaciones de aprendizaje en entornos virtuales. De hecho, según indican estudios sobre las interacciones entre pares en contextos virtuales, la habilidad y la competencia de la gente para trabajar efectivamente en grupo resulta central (Aragon, 2003; Cobb, 2009; Garrison, Anderson y Archer, 2000 y 2003; Mycota y Duncan, 2007; Wheeler, 2005).

Poniendo énfasis también en la importancia de atender a la dimensión social de las interacciones, diversos estudios afirman la importancia de la regulación de emociones y de la motivación en situaciones de trabajo colaborativo, así como el logro de la regulación social del aprendizaje (López Benavides, 2009; Järvelä, Hurme y Järvejona, 2009; Järvenjona, 2010; Rendón, 2010). Efectivamente, en experiencias que hemos llevado adelante en nuestro equipo, se apreciaron recurrentemente funcionamientos grupales que difieren de manera notoria y que reflejan diferentes niveles de habilidad de los sujetos para manejarse en un entorno menos familiar que el del trabajo cara a cara (Chiecher y Paoloni, 2011; Chiecher y Donolo, 2011).

Siguiendo esta línea de trabajos, interesa en este artículo tomar posición en una perspectiva social, centrando la atención en las relaciones interpersonales dentro de los grupos así como en las elecciones y rechazos manifestados por los alumnos en relación con sus pares. 


\section{MÉTODO}

Se trabajó en el marco de la propuesta metodológica de los estudios de diseño (Rinaudo y Donolo, 2010) ${ }^{1}$. En ese contexto, se implementó con diferentes grupos de alumnos un diseño instructivo, que proponía dar respuesta -en pequeños grupos y mediante intercambios virtuales- a una tarea académica.

Sujetos. Participaron del estudio 154 sujetos; 89 de ellos cursaban estudios de grado en la Universidad Nacional de Río Cuarto (Argentina). Los restantes 65 cursaban estudios de posgrado en el marco de un curso de formación dictado por la Universidad Nacional de Jujuy (Argentina) y un Doctorado en Enseñanza de las Ciencias Exactas y Naturales dictado por la Universidad Nacional de Comahue (Argentina).

Los grupos de grado y de posgrado diferían en cuanto a edad (los alumnos de grado eran más jóvenes que los de posgrado); género (los alumnos de grado eran en su mayoría mujeres en tanto que los de posgrado mantenían proporciones similares de varones y mujeres) y responsabilidades paralelas al estudio (los alumnos de grado no tenían, en general, otra responsabilidad más allá del estudio, en tanto que los de posgrado atendían simultáneamente a responsabilidades laborales y familiares).

Los 154 sujetos se distribuyeron en 36 grupos -22 conformados por estudiantes de grado y 14 integrados por alumnos de posgrado- que son las unidades de análisis en las que focalizará el presente artículo.

Instrumentos. Con el propósito de conocer la estructura, las relaciones y las elecciones y rechazos dentro de los grupos virtuales se diseñó y administró un cuestionario sociométrico elaborado sobre la base del instrumento que presentan García-Bacete y González (2010).

El cuestionario contiene seis preguntas ${ }^{2}$, de las que se tomaron para el análisis presentado en este artículo aquellas que indagan sobre los motivos de elección y rechazo entre pares. Precisamente, las preguntas cuyas respuestas se analizan, solicitan al sujeto realizar elecciones positivas (pregunta A) y nominaciones negativas de sus compañeros de grupo (pregunta B), fundamentando en cada caso las razones de tales decisiones. 


\section{PROCEDIMIENTO}

Con cada curso se trabajó en el escenario natural de dictado de la asignatura. En este marco, se propuso la resolución de una actividad -en cada caso vinculada al contenido del curso- que los estudiantes debían resolver conformados en grupos y con la mediación de un entorno virtual (foros).

Los grupos trabajaron conjuntamente e interactuaron en el espacio virtual entre 2 y 4 semanas. Hacia el final de la actividad, una vez que los alumnos habían tenido ya la experiencia de trabajar en interacción virtual con sus compañeros, se administró el cuestionario sociométrico mediante el que se recogieron los datos del presente estudio. El instrumento se puso a disposición en el aula virtual, en un archivo de formato Word, para que los estudiantes pudieran descargarlo, responderlo y subirlo completo en la misma plataforma.

\section{RESULTADOS}

\section{Caracterización de los grupos de grado y posgrado}

Se trabajó con un total de 36 grupos -conformados por 2 a 7 estudiantes- que, en el marco de distintos cursos, daban solución a una determinada tarea, manteniendo para ello intercambios en un entorno virtual; 22 grupos estaban conformados por estudiantes que cursaban carreras de grado, a quienes se agrupó al azar y por designación de los profesores. Otros 14 grupos, estaban conformados por estudiantes de nivel de posgrado, quienes decidieron voluntariamente trabajar juntos con el objetivo de dar solución a una actividad en el marco del curso.

Además de ser diferentes en cuanto al criterio de formación, los grupos eran disímiles también en cuanto a algunas de sus características básicas a las que hacemos referencia a continuación.

En cuanto al tamaño de los grupos, aquellos integrados por estudiantes de grado eran en su mayoría de 4 alumnos (50\% de los grupos); en menor medida grupos de 5 alumnos (27\%) o bien 3 alumnos (23\%). En cambio, los grupos de alumnos de posgrado fueron más variados en cuanto a cantidad de integrantes (variaron entre 2 y 7) y tendían a ser más numerosos; así, 36\% de los grupos eran de 5 integrantes; $21 \%$ de 4 alumnos; $14 \%$ de 7 integrantes; $14 \%$ de 3 alumnos y $7 \%$ de solo 2 integrantes.

Considerando el género de los integrantes de cada grupo, se observan grupos de composición enteramente femenina y otros de composición mixta. En este sentido, se aprecian notorias diferencias entre los grupos de grado, en su mayoría de conformación exclusivamente femenina (91\%), y los alumnos de posgrado cuya situación se presenta inversa (83\% de grupos de conformación mixta). 
También diferían los grupos en cuanto a la edad y dedicación al estudio; los grupos de grado estaban conformados por alumnos jóvenes en tanto que los grupos de posgrado integraban adultos. En general, los jóvenes (estudiantes de grado) dedicaban su tiempo exclusivamente al estudio mientras que los adultos (estudiantes de posgrado) compartían la dedicación al estudio con responsabilidades laborales y familiares.

Si bien las diferencias mencionadas podrían incidir en el tipo de relaciones y en la dinámica de los grupos, veremos a continuación que los resultados no se presentaron muy disímiles en los grupos de grado y de posgrado. Esto es, pareciera que el tamaño de los grupos, la composición mixta o no, la posibilidad de elegirse o el tener que trabajar forzosamente con otros, etc., en este caso, no fueron variables que hayan tenido alto impacto en las elecciones y motivos que dieron los estudiantes para elegir o rechazar a un compañero.

\section{Grupos tipo en grado y posgrado}

Sobre la base del cálculo del Modo (o categoría más frecuentemente observada) de ciertas variables (cantidad de integrantes en el grupo, composición según género, cantidad de elecciones positivas emitidas en el grupo, cantidad de elecciones negativas emitidas en el grupo, cantidad de elecciones positivas recíprocas y cantidad de elecciones negativas recíprocas) es posible detectar y construir las características del grupo tipo o más frecuente.

\section{Tabla 1.}

Modo de variables estructurales de 36 grupos de grado y posgrado

\begin{tabular}{|l|c|c|}
\hline Cantidad de integrantes (Modo) & $\begin{array}{c}\text { Grupos } \\
\text { de grado } \\
(\mathrm{N}=22)\end{array}$ & $\begin{array}{c}\text { Grupos de } \\
\text { posgrado } \\
(\mathrm{N}=14)\end{array}$ \\
\hline Composición según género (Modo) & femenina & 5 alumnos \\
\hline Elecciones positivas emitidas (Modo) & 2 & 2 \\
\hline Elecciones negativas emitidas (Modo) & 0 & 0 \\
\hline Reciprocidades positivas (Modo) & 1 & 0 \\
\hline Reciprocidades negativas (Modo) & 0 & 0 \\
\hline
\end{tabular}

Conforme al análisis de los modos registrados en cada variable, el grupo típico entre los estudiantes de grado está conformado por 4 integrantes, es exclusivamente femenino en su composición, registra 4 elecciones positivas, una de ellas recíproca (un alumno elige a un compañero y al mismo tiempo es elegido por este) y ninguna 
nominación negativa o rechazo. Gráficamente, podría representarse del siguiente modo.

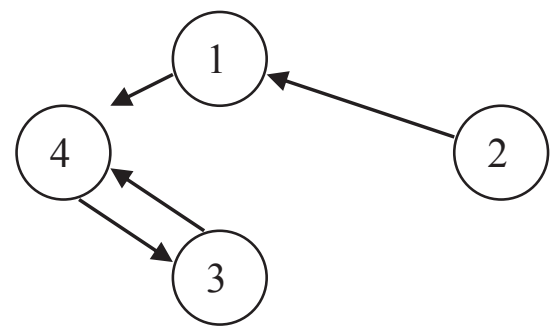

Figura 1. Sociograma ilustrativo de grupo típico de grado

Por su parte, el grupo típico de alumnos de posgrado está conformado por 5 integrantes, es mixto en su composición (incluye varones y mujeres), registra 2 elecciones positivas, ningún rechazo y ninguna reciprocidad (sea positiva o negativa). Gráficamente puede representarse del siguiente modo.

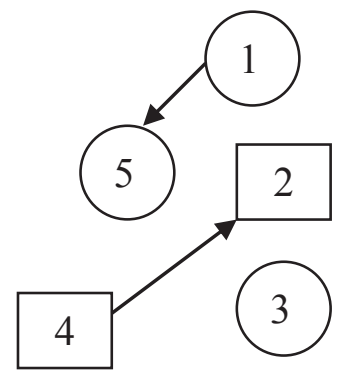

Figura 2. Sociograma ilustrativo de grupo típico de posgrado

Tal como se infiere conforme a la información presentada, los grupos no manifestaron, en la mayoría de los casos, problemas serios en su funcionamiento. En principio, los grupos típicos en grado y posgrado no registraron elecciones negativas o de rechazo hacia el compañero. Tampoco puede hablarse, no obstante, de un alto nivel de integración en los equipos de trabajo puesto que en los grupos de posgrado estuvieron ausentes las reciprocidades positivas y en los de grado lo más frecuente fue observar solo una elección recíproca positiva.

Otra cuestión a destacar es que los estudiantes de grado, aún cuando fueron forzados a trabajar con compañeros a quienes en principio no hubieran elegido, estuvieron más dispuestos a nominar positivamente; de hecho, el 89\% de los alumnos de grado realizó una o más nominaciones positivas. En cambio, entre los sujetos de 
los grupos de posgrado -quienes se auto asignaron a los diferentes grupos, eligiendo por lo tanto a sus compañeros- el porcentaje de quienes realizaron nominaciones positivas resulta algo inferior, representando un $80 \%$ (como vemos, en el grupo típico, más de un integrante no emite nominaciones).

Más reacios aún se mostraron a realizar nominaciones negativas. Solamente un $24 \%$ y un $23 \%$ de los alumnos de grado y posgrado respectivamente realizaron alguna. Tal como se aprecia en los gráficos, los grupos típicos se caracterizan por la ausencia de nominaciones negativas o rechazos.

\section{Elecciones positivas y negativas en los grupos de grado y de posgrado}

Para hacer comparables los grupos -puesto que como vimos difieren en cuanto a cantidad de integrantes- se calculó el índice de nominaciones positivas recibidas grupal (IndNPRg) y el índice de nominaciones negativas recibidas grupal (IndNPRg) siguiendo la fórmula propuesta por García-Bacete y González (2010). El valor de este índice representa el número de nominaciones positivas y negativas que recibiría cada alumno del grupo si las nominaciones se distribuyeran de forma equitativa entre todos los compañeros.

La Tabla 2 muestra medidas de tendencia central para las variables mencionadas.

Tabla 2.

Media, modo, valor máximo y mínimo de IndNPRg y IndNNRg

\begin{tabular}{|l|c|c|}
\hline Media de IndNPRg & $\begin{array}{c}\text { Grupos de grado } \\
(\mathrm{N}=22)\end{array}$ & $\begin{array}{c}\text { Grupos de } \\
\text { posgrado }(\mathrm{N}=14)\end{array}$ \\
\hline Modo de IndNPRg & 1.16 & 1.34 \\
\hline Mínimo y Máximo de IndNPRg & 1 & 1 \\
\hline Media de IndNNRg & $.33-2.40$ & $.00-3.20$ \\
\hline Modo de IndNNRg & .25 & .24 \\
Mínimo y Máximo de IndNNRg & 0 & 0 \\
\hline
\end{tabular}

Tal como se aprecia, tanto en grado como en posgrado son más altas las medias de los IndNPRg en comparación con las de los IndNNRg. Tanto en los grupos de grado como de posgrado, el modo o situación más frecuente, fue una (1) elección positiva de parte de cada sujeto.

Por su parte, las medias de los IndNNRg, tanto para grupos de grado como de posgrado, fueron inferiores a las de los IndNPRg. En ambos casos el Modo, o 
situación más frecuentemente observada, indica cero (o) rechazo por parte de cada sujeto. En efecto, en 16 de los 36 grupos (44\%) no se observaron rechazos, lo que podría indicar un mayor nivel de integración en estos grupos.

Las figuras que siguen presentan la cuestión de la cantidad de elecciones positivas y negativas de manera más detallada.

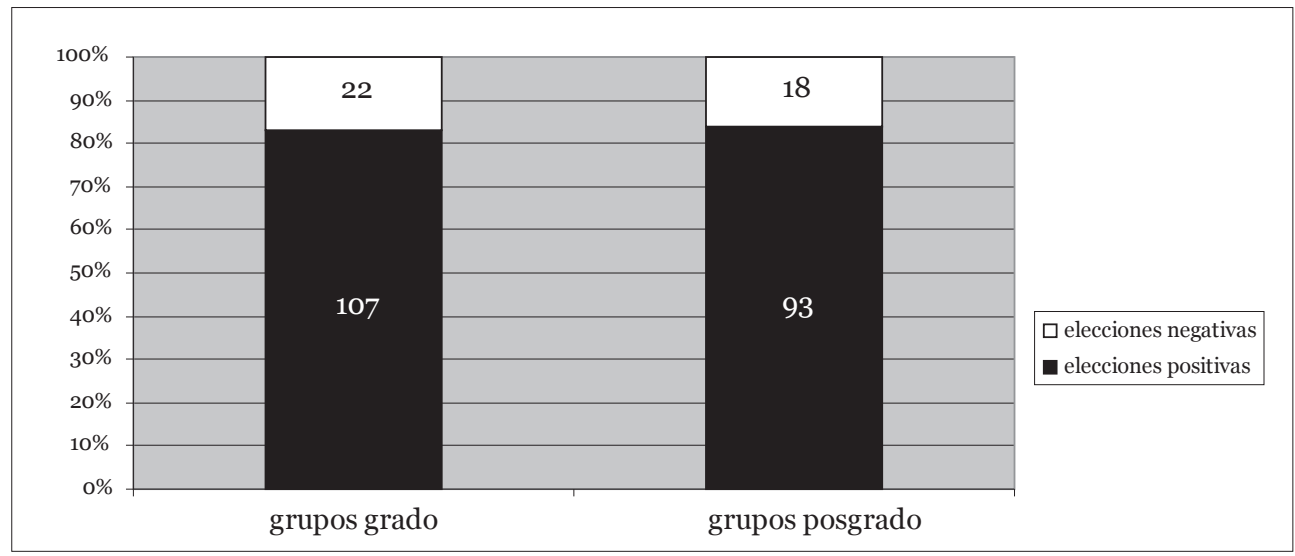

Figura 3. Cantidad de elecciones positivas y negativas en grupos de grado y posgrado

En general, fueron mayoritarias las elecciones positivas y se presentaron con una distribución similar en los grupos de grado y de posgrado.

Como muestra el gráfico, el 83\% (107) y 84\% (93) de las elecciones dentro de los grupos de grado y posgrado respectivamente fueron positivas, incluyendo además un importante número de elecciones positivas que fueron recíprocas.

Contrariamente, una menor proporción está conformada por elecciones negativas o de rechazo hacia alguno de los integrantes del grupo (17\% en los grupos de grado y $16 \%$ en los de posgrado).

\section{Motivos de elección positiva}

Interesa conocer los motivos o razones por las que un sujeto realiza una elección o valoración positiva del trabajo de un compañero, pues ello puede ofrecer pistas al momento de considerar criterios para formar grupos de trabajo.

Con este fin se analizaron los motivos declarados por los sujetos, conformándose como consecuencia del análisis seis categorías de respuesta no mutuamente 
excluyentes; esto es, la respuesta de un sujeto podía aludir a más de un motivo o razón.

Cabe señalar que, más allá de las diferencias iniciales entre los grupos de grado y posgrado, los motivos de elección positiva fueron similares en ambas poblaciones.

Las categorías emergentes a partir de las respuestas de los estudiantes de grado y de posgrado fueron las siguientes:

- Cualidades personales positivas del compañero. Se incluyen en esta categoría todos aquellos argumentos que aluden a características de personalidad o cualidades destacadas de un compañero, entre ellos, el referir al otro como una persona agradable, sencilla, responsable, respetuosa, atenta, productiva, resolutiva, que sabe escuchar, abierta, capaz, espontánea, paciente, etc. (Ejemplo: elegí a tal compañera porque "... es muy estudiosa, meticulosa, responsable, respetuosa y consciente de la tarea grupal').

- Sintonía en el modo de trabajar. En esta categoría se incluyen razones que señalan sintonía o compatibilidad con el compañero elegido, específicamente en la forma de trabajar. (Ejemplos: elegí a tal compañera porque “... considero que es con la que más comparto la forma de trabajar").

- Compromiso con la tarea. Esta categoría comprende aquellas respuestas que aludieron específicamente al compromiso asumido por el compañero en la resolución de la tarea que tenían que resolver. (Ejemplo: elegí a tal porque “... tiene un gran sentido de compromiso con la actividad").

- Experiencias previas de trabajo conjunto. Se incluyen aquí argumentos que avalan la elección de determinado compañero por haber compartido previamente experiencias de trabajo conjunto. (Ejemplo: elegí a tal porque “... he trabajado con ella anteriormente en otras materias").

- Afinidad personal con el compañero. La categoría incluye expresiones que aluden a una relación de afinidad con el compañero elegido; relación que puede haber sido construida con anterioridad al trabajo grupal propuesto o bien haberse logrado en esa instancia. (Ejemplo: elijo a tal porque “... además de ser mi compañera de la carrera es mi amiga y es con quien habitualmente tenemos una muy buena comunicación”).

En cuanto a la distribución de las respuestas, la figura 4 muestra la frecuencia de presentación de cada una de las categorías en los grupos de grado y de posgrado. 


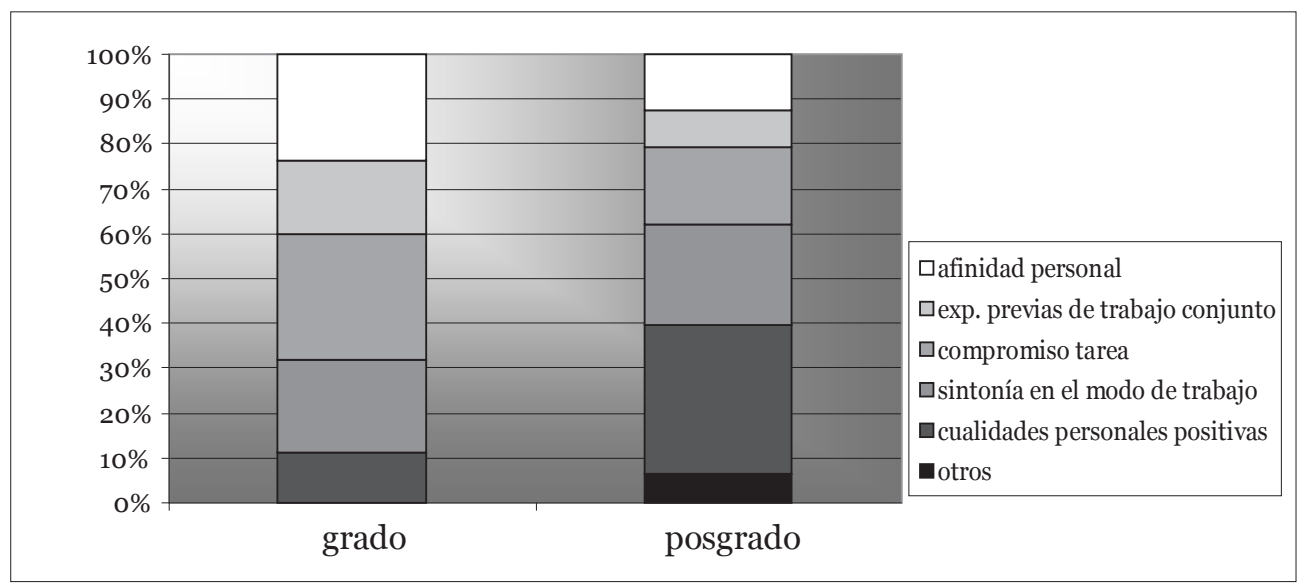

Figura 4. Motivos de elección positiva en grado y posgrado

En los grupos de grado, el motivo principal para elegir a un compañero fue el compromiso asumido con la tarea que tenían que realizar; 27 sujetos aludieron a esta razón como fundamento de su elección; con una frecuencia similar se aludió a la afinidad con la persona como motivo de la elección positiva (23 sujetos) y a la sintonía en el modo de trabajo (20 sujetos). En orden decreciente, con 16 menciones, se ofrecieron argumentos correspondientes a la categoría de experiencias previas de trabajo conjunto y, finalmente, la categoría menos frecuente fue la que refiere a cualidades personales del compañero (11 sujetos).

Por su parte, entre los grupos de posgrado, los argumentos más frecuentes para justificar una elección positiva estuvieron relacionados con cualidades personales positivas del compañero (21 sujetos). Le siguen en orden de frecuencia, la sintonía en cuanto al modo de trabajo (14 sujetos); el compromiso con la tarea (11 sujetos); la afinidad personal (8 sujetos) y las experiencias previas de trabajo grupal (5 sujetos).

\section{Motivos de elección negativa o rechazo}

Respecto de las razones que fundamentan las elecciones negativas, fueron agrupadas en cuatro categorías de respuestas, algunas de ellas opuestas a las descritas en el apartado anterior. También en este caso, las categorías construidas resultan pertinentes para analizar las respuestas tanto de los grupos de grado como de posgrado.

Las categorías emergentes fueron las siguientes: 
- Nula afinidad con el compañero. En esta categoría se incluyeron respuestas que aludían a una nula afinidad personal, o relación distante, con el compañero rechazado. (Ejemplo: no elegiría a tal compañero porque “... no percibí que se generara un lazo de simpatía que me posicione para elegirlo y, a mi criterio, si se da la opción de elegir, jamás elegiría a alguien con el cual no hay una posibilidad de transparentar los sentimientos. Siento mucha distancia, en lo personal, entre ambos").

- Falta de compromiso con la tarea. Esta categoría incluye respuestas que fundamentan el rechazo de un compañero en función de su escasa participación y pobre compromiso con la tarea que debían realizar en conjunto. (Ejemplo: no elegiría a tal compañero porque "... sentí que estuvo un tanto ausente en la tarea").

- Diferencias en la modalidad de trabajo. La categoría comprende expresiones que fundamentan la elección negativa de un compañero en base a la incompatibilidad en cuanto al modo de trabajar para dar solución a la tarea propuesta. (Ejemplo: no elegiría a tal compañero porque “... no pude entender cómo es su forma de trabajar y de pensar").

- Características personales o actitudes negativas. Se incluyen en esta categoría todos aquellos argumentos que aluden a características de personalidad o actitudes negativas apreciadas en el compañero rechazado. Calificativos tales como poco agradable, mentiroso, egoísta, avasallante, de actitudes incorrectas, irresponsable, irrespetuoso, fueron usados por los sujetos para dar motivos de los rechazos hacia otros compañeros de grupo. (Ejemplo: no elegiría a tal compañero porque "... me parece una persona muy avasallante contra los demás, quiere realizar todo ella”).

La distribución de estos cuatro motivos de rechazo en los grupos de grado y posgrado es ilustrada en la figura 5 .

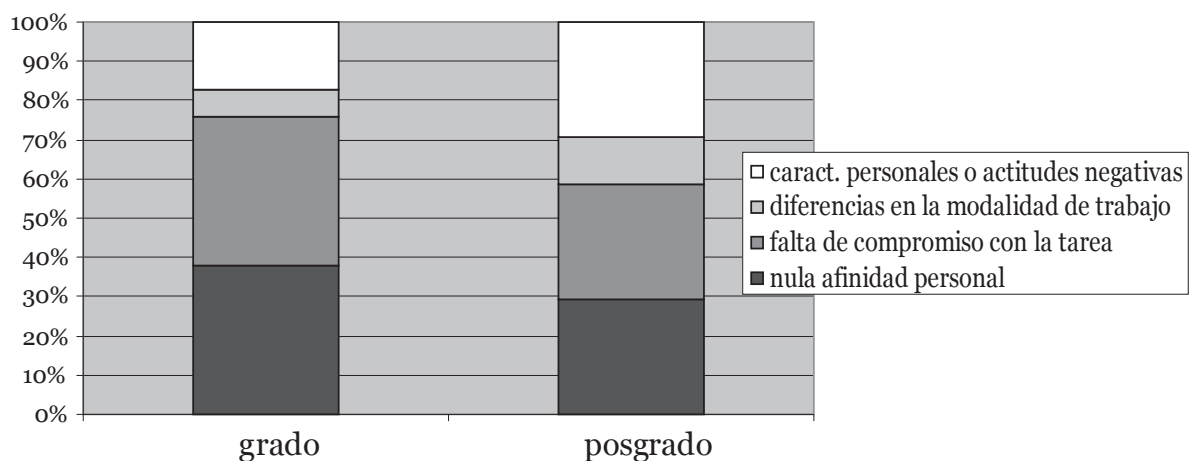

Figura 5. Motivos de elección negativa en grado y posgrado 
En los grupos de grado los motivos de rechazo relacionados con la falta de compromiso con la tarea por parte del compañero y la nula afinidad fueron los que explicaron la mayor parte de los rechazos (11 sujetos en cada caso mencionaron estos argumentos). En menor medida se registraron menciones de características personales o actitudes negativas del compañero rechazado (5 sujetos) y diferencias en la modalidad de trabajo (2 sujetos).

Por su parte, en los grupos de posgrado los motivos relacionados con cualidades personales negativas del compañero, afinidad nula y falta de compromiso se presentaron con igual frecuencia ( 5 sujetos los mencionaron en cada caso), en tanto que fue algo menor la participación de motivos relacionados con las diferencias en la modalidad de trabajo (dos menciones en cada grupo).

\section{DISCUSIÓN Y CONCLUSIONES}

Como decíamos al inicio, si bien los efectos positivos del trabajo en grupos han sido estudiados y probados, no se producen de manera espontánea con solo formar grupos de alumnos. Es necesario atender al funcionamiento del proceso de interacción y a los principales mecanismos responsables de su eficacia para poder apoyarlo, regularlo y estructurarlo debidamente (López Benavides, 2009). Más aún en entornos virtuales, donde los mecanismos y la situación misma de interacción difieren de las más habituales situaciones de trabajo grupal en entornos presenciales.

Se trata, en efecto, de un campo de estudio muy complejo, en el que se entrelazan importantes y grandes temas como el de la autorregulación del aprendizaje, la regulación y activación de emociones frente a los comportamientos y actitudes del otro, la motivación, las habilidades de comunicación en entornos asincrónicos y por medio del lenguaje escrito, entre otros.

En el presente estudio se trabajó con grupos de alumnos de grado y de posgrado, inicialmente de características distintas. No obstante las diferencias señaladas, los resultados mostraron ciertas similitudes en cuanto a las relaciones interpersonales desplegadas y los motivos ofrecidos para justificar las elecciones o rechazos de un par $^{3}$. En efecto, tanto entre los alumnos de grado como de posgrado, se registró la mayoría de elecciones o nominaciones positivas de los compañeros, avaladas por motivos que aluden a las cualidades personales del otro, a la sintonía en el modo de trabajo, a la afinidad personal, a las experiencias previas en trabajos compartidos y al compromiso asumido con la tarea. Paralelamente, se observó un número notoriamente más reducido de nominaciones negativas, justificadas por motivos similares dentro de los grupos de grado y posgrado. Entre las razones de rechazo, tanto entre los alumnos de grado como de posgrado, figuraban las características personales o actitudes negativas del compañero, diferencias en la modalidad de trabajo, falta de compromiso con la tarea y nula afinidad personal. 
Si bien más escasas, la presencia de nominaciones negativas en varios grupos así como los motivos que las justifican, se convierten en potenciales generadoras de conflictos y de funcionamientos deficientes dentro de los ellos. De ahí, la importancia de atender particularmente a estas relaciones teñidas por el rechazo y a los motivos que las generan.

Por fin, más allá de recapitular aquí algunos de los principales resultados hallados en el estudio de los motivos de elección y rechazo entre pares en situaciones de trabajo grupal y virtual, nos orienta el interés más ambicioso de vincular los resultados presentados con planteos teóricos referidos a la regulación compartida del aprendizaje en entornos virtuales. Nos atrevemos también, en el último apartado, a sugerir acciones concretas orientadas a favorecer el logro de esta regulación social o compartida dentro de grupos que funcionan en escenarios virtuales. A estas dos cuestiones están dedicados los siguientes apartados.

\section{Hacia el logro de la co-regulación o regulación compartida del aprendizaje en entornos virtuales}

Una diferencia sustancial marca una línea divisoria importante entre las situaciones de trabajo grupal en contextos presenciales y virtuales. El trabajo en grupos en entornos presenciales se desarrolla en co-presencia de los participantes, de manera sincrónica, siendo la comunicación entre los miembros del grupo predominantemente verbal $\mathrm{u}$ oral. Por el contrario, las situaciones de trabajo grupal en entornos virtuales se desarrollan, en general, con la mediación de recursos (el foro, por ejemplo) que admiten una comunicación asincrónica y diferida en el tiempo.

La novedad de la situación hace que sujetos probablemente hábiles en regular su aprendizaje y trabajar conjuntamente con otros en situaciones presenciales se vuelvan inexpertos o poco habilidosos para lograr un trabajo en equipo mediado tecnológicamente.

En este sentido, parece oportuno traer a colación la idea de que el trabajo en equipo demanda no solo la regulación individual del propio aprendizaje, sino también una importante dosis de regulación social, autorregulación grupal, coregulación o regulación compartida, en términos de distintos autores (Järvenoja, 2010; López Benavides, 2009; López y Álvarez, 2011; Mauri, Colomina y Gipert, 2009). No obstante, si la regulación individual del aprendizaje no es tarea sencilla, menos aún lo es conducir el proceso en situaciones en las que se debe construir conocimientos junto con otros (Järvenoja, 2010).

Se tornan relevantes en estos casos las conductas o comportamientos de negociación y de consideración del compañero, de sus posibilidades, limitaciones, de sus avances y dificultades durante el proceso de aprendizaje. En tal sentido, 
parece clave -entre otras cosas- lograr una planificación consensuada de la actividad a resolver y de los pasos a seguir, de los tiempos disponibles para participar, tratar de cumplir lo pactado en los acuerdos iniciales, conectarse con frecuencia y asiduidad para ver las opiniones de los compañeros, dejar constancia y aviso de lo que cada uno está realizando, revisar exhaustivamente el requerimiento de la actividad a desarrollar, etc. (López Benavides, 2009). En otros términos, se tornan cruciales las competencias, sobre todo sociales y participativas, definidas como un conjunto de conocimientos, capacidades, aptitudes y habilidades necesarias para realizar actividades diversas con cierto nivel de calidad y eficacia (Iglesias, 2009).

Como vimos, la falta de compromiso con la tarea fue un importante motivo de rechazo y sospechamos que puede relacionarse con conductas poco habilidosas para atender a la regulación social del aprendizaje. Así, puede que un sujeto tenga intención de contribuir a la resolución grupal de una actividad pero no se percate, sin embargo, de la importancia de conectarse asiduamente para estar al tanto del proceso grupal, de la relevancia de leer los aportes de los compañeros tratando de partir de allí para seguir avanzando, o de la trascendencia de avisar que se está avanzando en tal sentido y que no será posible conectarse por determinadas razones. Esto es, el trabajo conjunto requiere un esfuerzo de coordinación, en el sentido de que cada uno debería saber qué está haciendo el otro, hacia dónde puede avanzar y qué tipo de ayuda necesita en caso de tener dificultades.

Ahora bien, aún cuando parece esencial la influencia de la calidad de la regulación social durante la resolución de tareas cooperativas, no se puede perder de vista la participación de variables personales que también pueden explicar numerosas dificultades de coordinación, de ambigüedad en papeles, de conflictos personales, etc., y que hacen que los resultados del trabajo del grupo no sean los esperados (López Benavides, 2009). En esta línea, características o comportamientos personales negativos, tales como los que fueron mencionados por los estudiantes para explicar el rechazo de un compañero (egoísmo, irresponsabilidad, irrespetuosidad, soberbia, etc.), parecen más difíciles de poder modificar desde afuera en pos de lograr un mejor funcionamiento grupal.

\section{Ayudas externas para contribuir al logro de la regulación social dentro de los grupos}

Quisiéramos cerrar el artículo destacando la posibilidad de intervenir externamente, desde el rol docente, para contribuir al logro de una dinámica positiva dentro de los grupos. De hecho, el profesorado desempeña un rol crucial en la adopción e implementación de las TIC en la educación (Almercih, Suárez, Orellana y Díaz, 2010). Si bien, en cuanto a las variables personales, es más difícil poder intervenir, sí parece posible incidir sobre algunas otras variables, promoviendo el 
aprendizaje de habilidades para desenvolverse apropiadamente en el marco del trabajo colaborativo en entornos virtuales.

Entre las estrategias que contribuirían a apoyar y organizar el trabajo dentro de los grupos están: el monitorear permanentemente el trabajo que llevan adelante; mediar en los conflictos cuando resulte posible, contactar a los alumnos que no participan, invitándolos a hacerlo e intentando conocer las razones de su ausencia, ofrecer feedback acerca de la marcha del proceso; estructurar temporalmente las actividades calendarizando sus distintas instancias o etapas, etc.

\section{NOTAS}

1. Más detalle acerca de los estudios de diseño pueden consultarse en Rinaudo y Donolo (2010), Estudios de diseño. Una perspectiva prometedora en investigación educativa. Revista RED, 22. Recuperado de http://www.um.es/ead/red/22/rinaudo donolo.pdf

2. Si bien el instrumento consta de 6 preguntas o ítems que el sujeto debía responder, dos de ellas (A y B) son las que se vinculan específicamente con el tema que tratamos en el presente artículo. Por un lado, el ítem que solicita nominación de uno (o más compañeros) a quien/es el sujeto elegiría para trabajar en grupo así como la explicitación de los motivos de esa elección. Por otro lado, la nominación negativa; es decir, la mención de compañeros con quienes no volverían a trabajar y la consecuente explicitación de los motivos que avalan tal decisión. Dentro de las preguntas o ítems no analizados, dos de ellas solicitan al sujeto expresar sus percepciones o creencias acerca de quiénes creen que podrían elegirlos (C) y quiénes no los elegirían para el trabajo en grupos (D). Por fin, las últimas dos preguntas solicitan un ordenamiento de los miembros del grupo conforme a la apreciación de sus aportes en el trabajo grupal (E) y la mención de los compañeros con quienes habitualmente trabajan, cuando los grupos se forman espontáneamente $(\mathrm{F})$. Entendemos que en futuras publicaciones será posible atender a un análisis complementario del aquí presentado, focalizando en las respuestas consignadas para otros ítems.

3. El hecho de observar similitudes entre los grupos de grado y posgrado en los resultados referidos a los motivos de elección y rechazo entre pares adquiere mayor sentido, quizás, considerado en un contexto más amplio. En efecto, en otros estudios hemos observado reiteradamente diferencias notorias entre grupos de estudiantes de grado y de posgrado. Por ejemplo, en los perfiles motivacionales y cognitivos (Chiecher et al., 2009a), en el manejo y la planificación del tiempo y el ambiente (Chiecher, 2009), en la búsqueda de ayuda (Chiecher et al., 2009b), entre otros. 


\section{REFERENCIAS BIBLIOGRÁFICAS}

Almerich, G., Suárez, J., Orellana, N., y M. I. Díaz. (2010). La relación entre la integración de las tecnologías de la información y la comunicación y su conocimiento. Revista de Investigación Educativa, 28(1), 31-50.

Aragon, S. (2003). Creating social presence in online environments. New Directions for Adult and Continuing Education, 100, 57-68.

Chiecher, A. (2009). Gestión del tiempo y el ambiente de estudio en cursos online. Estudio comparativo de tres grupos de estudiantes de diferente perfil. Memorias I Congreso Internacional de Investigación y Práctica Profesional en Psicología. Tomo I. Buenos Aires: Universidad de Buenos Aires.

Chiecher, A., Donolo, D., Rinaudo, M. C., Cabello, R., y Asaad, C. (2009a). Enseñar y aprender. Motivación, estrategias $y$ percepción del contexto en entornos presenciales y virtuales. Río Cuarto: EFUNARC.

Chiecher, A., Donolo, D., y Rinaudo, M. C. (2009b). Búsqueda de ayuda en foros electrónicos. Frecuencia y contenido de las preguntas formuladas por estudiantes de grado y posgrado. Revista Iberoamericana de Educación a Distancia, 12 (2), 139-161.

Chiecher, A., y Paoloni, P. (2011). Trabajo en equipo en contextos de educación a distancia mediados por TIC: una propuesta para medir la dimensión social en grupos virtuales. XI Coloquio Internacional de Gestión Universitaria de América del Sur. Universidad de Santa Catarina, Florianópolis, Brasil.

Chiecher, A., y Donolo, D. (2011). Innovar en la enseñanza de grado. Crónica de una experiencia de trabajo grupal en entornos virtuales. RED, Revista de Educación a Distancia, 29, 1-19.

Cobb, S. (2009). Social presence and online learning: a current view from a research perspective. Journal of Interactive Online Learning, 8(3), 241-254.

De Jong, T., y Pieters, J. (2006). The design of powerful learning environments. En P. Alexander, y P. Winne, (Eds.). Handbook of Educational Psychology, (pp. 739754). Mahwah: Lawrence Erlbaum.

Fernández Prado, J. S. (2000). Sociología de los grupos escolares: sociometría y dinámica de grupos. Recuperado de http://www2.uah.es/conchacarrasco/ materiales/sociometria.pdf

García-Bacete, F. J., y González, J. (2010). Evaluación de la competencia social entre iguales. La sociometría y otras medidas. Madrid: TEA.

Garrison, R., Anderson, T., y Archer, W. (2000). Critical inquiry in a text-based environment: computer conferencing in higher education. The Internet and Higher Education, 2, 87-105.

Garrison, R., Anderson, T., y Archer, W. (2003). A theory of critical inquiry in online distance education. En M. Moore, y T. Anderson, (Eds.). Hanbook of Distance Education. (pp. 113-128). New Jersey: Lawrence Eilbaum Associates.

Iglesias Cortizas, M. T. (2009). Diagnóstico de las competencias emocionales: estudio empírico en la Facultad de Ciencias de la Educación de la Coruña. Revista de Investigación Educativa, 27 (2), 451-467.

Järvelä, S., Hurme, T., y Järvejona, H. (2009). Self-regulation and motivation in computer supported collaborative learning environments. Recuperado de $\quad$ http://lecotec.files.wordpress. com/2010/01/self-regulation-andmotivation-in-computer-supported1.pdf

Järvenoja, H. (2010). Socially shared regulation of motivation and emotions $y$ collaborative learning. University of Oulo. Recuperado de http:// herkules.oulu.fi/isbn9789514263309/ isbn9789514263309.pdf 
López, D., y Álvarez, I. (2011). Promover la regulación del comportamiento en tareas de aprendizaje colaborativo en línea a través de la evaluación. RIED. Revista Iberoamericana de Educación a Distancia, 14, 161-183.

López Benavides, D. (2009). Regulación del comportamiento durante la construcción conjunta de conocimientos en tareas cooperativas en entornos de aprendizaje virtuales asincrónicos $y$ escritos. Universitat Oberta de Catalunya. Recuperado de http:// openaccess.uoc.edu/webapps/ o2/bitstream/10609/1921/1/ DTEPP 3 LopezDenise.pdf

Mauri, T., Colomina, R., y Gispert, I. (2009). Diseño de propuestas docentes con TIC para la enseñanza de la autorregulación en la Educación Superior. Revista de Educación, 348, 377-399.

Mycota, D., y Duncan, R. (2007). Learner characteristics as predictors of online social presence. Canadian Journal of Education, 30, 155-170.

Rendón, M. I. (2010). Regulación emocional y programas de aprendizaje socioemocional. Típica, Boletín de Salud Escolar, 6(1), 193-209.

Rinaudo, M. C. (en prensa). Estudios sobre los contextos de aprendizaje: arenas y fronteras. En P. Paoloni, M. C. Rinaudo, y A. González Fernández, (comps.). Cuestiones en Psicología Educacional. Perspectivas teóricas, metodológicas y estudios de campo. Sociedad Latinoamericana de Comunicación Social: España.

Rinaudo, M. C., y Donolo, D. (2010). Estudios de diseño. Una perspectiva prometedora en investigación educativa. RED. Revista de Educación a Distancia, 22, 1-19.

Wheeler, S. (2005). Creating social presence in digital learning environments: a presence of mind? Recuperado de http://videolinq.tafe.net/learning2005/ papers/wheeler.pdf

\section{PERFIL ACADÉMICO Y PROFESIONAL DE LA AUTORA}

Analía Chiecher. Doctora en Psicología por la Universidad Nacional de San Luis; Magíster en Educación y Universidad y Licenciada en Psicopedagogía por la Universidad Nacional de Río Cuarto. Investigadora adjunta de CONICET. Autora de artículos y libros sobre aprendizaje en ambientes presenciales y virtuales.

E-mail: achiecher@hotmail.com

DIRECCIÓN DE LA AUTORA:

Juan B. Justo 925,

Río Cuarto (5800) Argentina.

Fechas de recepción del artículo: 24/07/14

Fecha de aceptación del artículo: 11/09/14

\section{Como citar este artículo:}

Chiecher Costa, A. C. (2015). Elecciones y rechazos entre pares en situaciones de trabajo grupal mediado tecnológicamente. RIED. Revista Iberoamericana de educación a Distancia, volumen 18, $\mathrm{n}^{0}$ 1, pp. 213-229. 